\title{
Investigating the structural changes due to adenosine methylation of the Kaposi's sarcoma-associated herpes virus ORF50 transcript
}

\author{
Konstantin Röder ${ }^{1, \uparrow}$, Amy M. Barker ${ }^{2}$, Adrian Whitehouse ${ }^{2}$, and Samuela Pasquali ${ }^{3, \star}$ \\ ${ }^{1}$ Yusuf Hamied Department of Chemistry, University of Cambridge, Cambridge, CB2 1EW, UK \\ ${ }^{2}$ School of Molecular and Cellular Biology and Astbury Centre of Structural Biology, University of Leeds, Leeds, UK \\ ${ }^{3}$ Laboratoire CiTCoM, UMR 8038 CNRS, and Laboratoire BFA, UMR 8251 CNRS, Université de Paris, France
}

\begin{abstract}
Kaposi's sarcoma-associated herpes virus (KSHV) is a human oncovirus. KSHV relies on manipulating the host cell N6methyl adenosine (m6A) RNA modification pathway. Methylation within a RNA stem loop of the open reading frame 50 (ORF50) enhances transcript stability via the recruitment of the m6A reader, SND1. In this contribution we explore the energy landscapes of the unmethylated and methylated RNA stem loops of ORF50 to investigate the effect of methylation on the structure of the stem loop. We observe a significant shift upon methylation between an open and closed configuration of the top of the stem loop. In the unmethylated stem loop the closed configuration is much lower in energy, and, as a result, exhibits higher occupancy.
\end{abstract}

Correspondence: ${ }^{\dagger}$ Email: kr366@cam.ac.uk,

* Email: samuela.pasquali@parisdescartes.fr

\section{Introduction}

Kaposi's sarcoma-associated herpes virus (KSHV) is a human oncovirus associated with Kaposi's sarcoma, a highly vascular tumor of endothelial lymphatic origin, and several other AIDS-associated malignancies (1). Like all herpesviruses, KSHV has a biphasic life cycle consisting of latent persistence and a lytic replication phase. Notably, both phases are required for KSHV-mediated tumorigenesis. Expression of a single KSHV-encoded protein, the replication and transcription activator (RTA) protein (2), is necessary and sufficient for the transition between latency and lytic replication, leading to the activation of the complete lytic cascade resulting in infectious virions.

RTA is encoded in the open reading frame 50 (ORF50) - and its expression is stimualted by various cellular cues such as plasma cell differentiation (3) and hypoxia (4). RTA activates transcription of lytic genes by directly interacting with RTAresponsive elements (RREs) found in multiple lytic gene promoters, or indirectly via interactions with cellular transcription factors, particularly RBP-J $\kappa$, AP- 1 , and Oct- 1 (2).

N6-methyladenosine $(\mathrm{m} 6 \mathrm{~A})$ is the most prevalent internal modification of eukaryotic messenger RNAs (mRNAs). Due to recent transcriptime-wide m6A mapping of multiple viruses, it is becoming evident that there is an interplay be- tween m6A-decorated viral RNA and the host cellular m6A machinery, resulting in modulation of viral replication output (5). Several studies have demonstrated the KHSV transcriptome is heavily m6A methylated (6). The m6A sites are targets for protein recognition by so-called $\mathrm{m} 6 \mathrm{~A}$ reader proteins, with different proteins leading to a variety of biological fates, from promotion of RNA degradation $(7,8)$ to enhancement of translation (9). In addition to direct recognition, indirect recognition is also possible via the so called m6A switch mechanism. Here, the methylation modification has been shown to destabilise and alter RNA structures allowing the recruitment of protein binding partners $(10,11)$.

In KSHV, m6A modification of the ORF50 RNA transcript leads to recruitment the m6A reader Staphylococcal nuclease domain-containing protein 1 (SND1) (6). SND1 binding to the ORF50 RNA stabilises the transcript, resulting in effective lytic replication. At the same time the binding is impaired upon depletion of the m6A methyltransferase, METT13, while depletion of SND1 results in destabilised RNA, lower levels of RTA and impaired lytic replication (6). m6A modification of the ORF50 transcript occurs at a classical DRACH, GGACU, motif situated in a 43mer hairpin. The hairpin is relatively unstable, due to a large number of unpaired nucleotides and resulting weak base pairing, and further destabilisation, likely associated with structural changes of the stem loop, is necessary for SND1 binding (6).

While it is likely that the m6A modification alters the RNA structure and as a result its binding affinity to SND1, it is not clear what structural change occurs, and how it allows the recruitment of a protein binding partner. The methylation will alter the structural ensembles accessible to the molecule and also impact the dynamic behaviour of the stem loop. As the structural heterogeneity displayed by RNA stem loops and the associated slow dynamics complicate experimental and computational studies, an enhanced sampling approach may yield additional insight into the structural and kinetic properties of the stem loop, and the effect of the methylation. This information may be obtained by an explicit exploration of the energy landscape of the unmethylated and methylated stem loop. While this approach has not previously been applied 
to study epigenetic changes, it has been successfully used to study mutations in a different stem loop (12), as well as other non-canonical nucleic acid structures, and is known to produce insight into structural ensembles and the transitions between them $(13,14)$.

Here, we present the free energy landscapes for the unmodified and the m6A modified stem loop within the ORF50 transcript. We observe two main structural ensembles, differentiated by the orientation of A22, the m6A modification site. In one ensemble, the nucleotide is inside the stem loop, not accessible to potential binding partners (in-configuration), while in the other ensemble, it is pointing away from the stem loop (out-configuration). While the unmodified RNA shows a strong preference for the in-configuration, the m6A modification allows for a higher population of the out-configuration, through structural alterations based on changes in the intricate pattern of stabilising interactions. We further observe a key change in the lower part of the stem loop, where a large bulge can also stabilise or destabilise the stem loop. Both processes are interconnected, and hence required for binding, as observed in experiment (6).

\section{Materials and Methods}

The energy landscapes of the unmodified and methylated stem loops were explored using the computational potential energy landscape framework $(13,14)$. Below we give a brief summary of the simulation techniques in this work; a detailed guide to exploring energy landscape in this way can be found elsewhere (15).

The two-dimensional structures predicted by Baquero-Perez et al. were used as starting points with three leading $\mathrm{U}$ nucleotides at the $5^{\prime}$ end, ${ }^{1}$ and initial three-dimensional structures were obtained from RNAComposer (16). RNAcomposer allows the translation of the two-dimensional into a three-dimensional structure via fragment assembly. This structure exhibits the correct canonical interactions, but will not necessarily be the optimal structure with respect to noncanonical interactions and flexible regions, such as bulges. Therefore, basin-hopping (BH) global optimisation (17-19) was used to obtain low energy structures using physical modelling, optimising these remaining interactions. Three sets of five $\mathrm{BH}$ runs were conducted, one set for the structure obtained from RNAComposer without the modification, a second set for the same structure with the m6A modification, and a third set starting from the unfolded structure. Each of the run consisted of $150,000 \mathrm{BH}$ steps, with grouprotation moves $(20,21)$ to create new configurations, and a convergence criterion for minima of $10^{-6} \mathrm{kcal} \mathrm{mol}^{-} 1 \AA^{-1}$. The 100 lowest energy minima from each run where used to seed the databases for the energy landscapes.

Discrete pathsampling $(22,23)$ was employed to create a kinetic transition network $(24,25)$. Transition state candidates were obtained using the double-nudged elastic band (DNEB) algorithm (26-28), and the actual transition states obtained

\footnotetext{
${ }^{1}$ This leading UUU motif means the full size of the simulated stem loop is 44 nucleotides, but we start the labelling at 0 to be consistent with previous work.
}

via hybrid eigenvector-following (29). We obtain the free energies using the superposition approach with a harmonic approximation (30). The rate constants are calculated using the new graph transformation (NGT) algorithm from the kinetic transition network $(30,31)$. We represent the energy landscapes as disconnectivity graphs $(32,33)$, which faithfully represent the topography of the energy landscape (i.e. the folding funnels and their substructure) and the energy scale. In these graphs, a structure is associated to a point on the vertical axis according to its free energy. A vertical line is drawn upwards from each point. Lines are merged when the energy of all transition states connecting the structures is exceeded, which is drawn in a discrete manner to allow proper visualisation. The resulting horizontal ordering is such that structures with easier transitions are closest, and therefore faithfully shows the funnel structure of the energy landscape.

Throughout, the ff99(34) force field with the Barcelona $\alpha / \gamma$ backbone modification(35) and the $\chi$ modification for $\operatorname{RNA}(36,37)$ was used with implicit solvent $(i g b=2)$. The parameters for the modified nucleotide were taken from the standard AMBER library (38).

Structures in the energy landscape are assigned to funnels and define structural ensembles. Low-energy structures in each ensemble are analysed using Barnaba software (39) to detect base pairs, stacking and to compute all torsions in the backbone, sugar and pucker. For each structure, the overall number of canonical, non-canonical and stacking interactions is recorded for each nucleotide. All these values are then averaged to give the average local behaviour of the ensemble.

\section{Results}

The key results of this study are the free energy landscapes for the unmodified and the 22m6A-modified RNA stem loops. Disconnectivity graphs $(32,33)$ for these two systems at $310 \mathrm{~K}$ are shown in Fig. 1 and Fig. 2, respectively. The three distinct structural ensembles associated with funnels are called $A, B$ and $C$ in the unmodified system, and the corresponding structural ensembles in the m6A-modified system are $A^{*}, B^{*}$ and $C^{*}$.

In both landscapes, we see a broad separation of the structures into configurations where A22 is pointing inwards (inconfiguration) and the nucleobase interacts with the surrounding nucleotides, and configurations where A22 is pointing away from the stem loop (out-configuration).

A significant difference is the relative energies of these structural ensembles. In both cases, the in-configuration is observed at the bottom of the free energy landscape. The outconfigurations in the unmodified stem loop are between 22 and $30 \mathrm{kcal} / \mathrm{mol}$ higher in free energy. In contrast to this large gap, the methylation significantly lowers this energy 


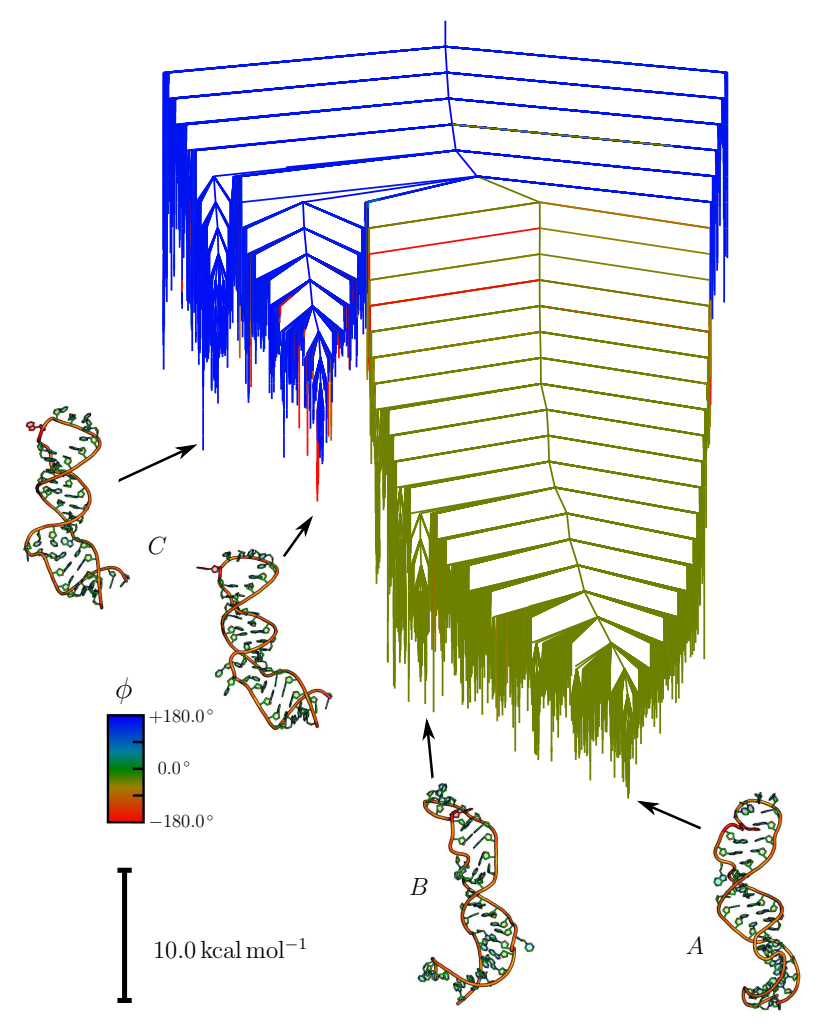

Fig. 1. Free energy disconnectivity graph for the native loop. The free energy disconnectivity graph at $310 \mathrm{~K}$ is shown for the unmethylated RNA stem loop. Two main funnels are observed, one where $\mathrm{A} 22$ is orientated inside the loop ( $A$ and $B$ ) and another where A22 is pointing outwards $(C)$. The former set of structures is much lower in free energy. The outwards orientation of A23 at the top of the stem loop is visible in the example structures shown on the graph for $C$, in contrast to the closed arrangement in $A$. The colouring scheme highlights the in/out-configuration of A22, and is based on $\phi$, which is the dihedral angle formed by the basepair below A22, and the nucleotide itself, where values around 0 indicate an in configuration (green), and values close to $\pm 180^{\circ}$ are out-configurations (blue and red). Some structural variation is observed in both major funnels, resulting in the emergence of smaller subfunnels. These variations are mainly located in the lower parts of the stem loop, leading to different stem configurations (see for example $A$ and $B$ ).

gap to around $10 \mathrm{kcal} / \mathrm{mol}$. In addition, multiple alternative stem configurations exist for the in-configuration, leading to substructure with some distinct subfunnels. For the unmodified system, these subfunnels are roughly 7 to $10 \mathrm{kcal} / \mathrm{mol}$ higher in free energy than the global minimum, while the m6A modification of A22 leads to more distinct substructure at slightly higher energies around 9 to $12 \mathrm{kcal} / \mathrm{mol}$. A full discussion of the important structural features is given later. It should be noted here that the energy of the local minimum for the structure obtained originally from RNAComposer is about $70 \mathrm{kcal} \mathrm{mol}^{-1}$ higher than the global minima for the unmodified and the modified system. There are two reasons for this observation. Firstly, the structure is in an out-configuration, and therefore already significantly higher in energy than the lowest energy minima in the $A$ and $A^{*}$ ensembles. Secondly, while the canonical base pairing is correct, due to the constraints from the two-dimensional structure, the non-canonical interactions and the flexible regions are not optimally arranged.

As a consequence of the change in relative energies of the structural ensembles, we predict a significant change in the

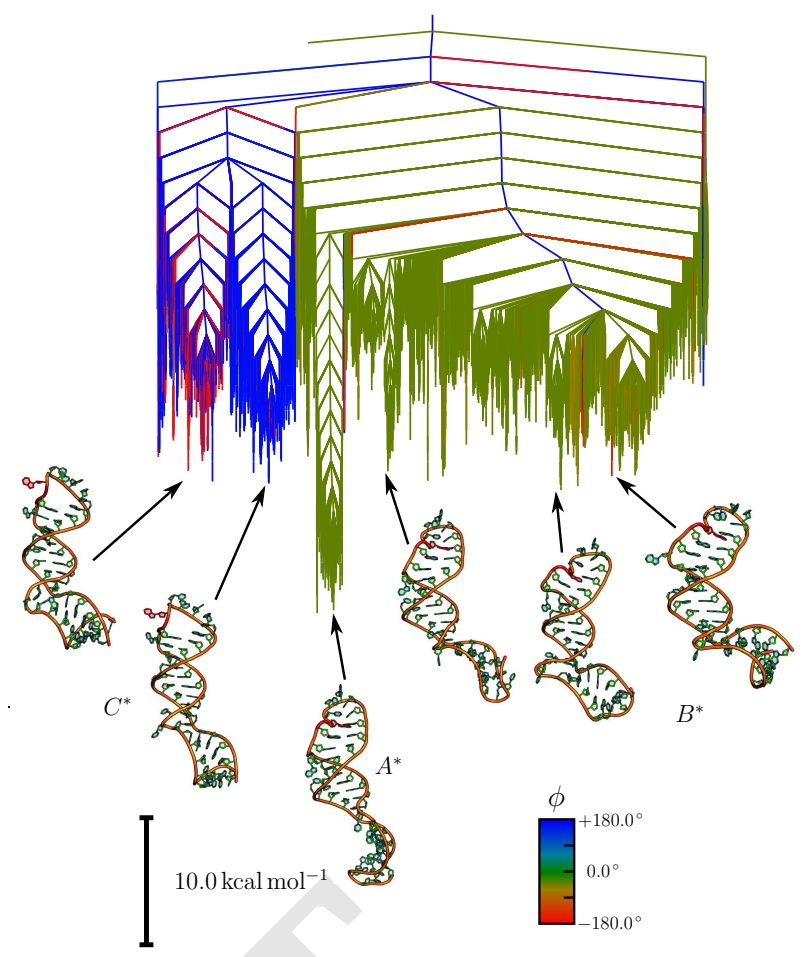

Fig. 2. Free energy disconnectivity graph for the modified stem loop. The free energy disconnectivity graph at $310 \mathrm{~K}$ for the methylated RNA stem loop exhibits significant changes compared to the unmethylated stem loop (see Fig. 1). The structural ensembles are preserved, but their relative energies are significantly altered. Firstly, the free energy of the out-configuration $\left(C^{*}\right)$ is lower, and in many cases comparable to the in-configurations. The set of lowest free energy structures is still an in-configuration $\left(A^{*}\right)$, but a large number of in-configurations $\left(B^{*}\right)$ are similar in energy to the out-configurations $\left(C^{*}\right)$, mainly due to changes in the lower part of the RNA stem loop.

transition rates between the different structures. All predicted rate constants and the associated equilibrium constants are given in Table 1. The transition from the into the out-configuration for A22 in the unmodified system is 10 orders of magnitudes slower than the reverse process $\left(1.535 \cdot 10^{-10} \mathrm{~s}^{-1}\right.$ vs. $\left.1.427 \mathrm{~s}^{-1}\right)$. Thus, we do not expect any significant population of the out-state at biologically relevant temperatures.

The picture for the m6A-modified stem loop is different, but also complicated by the topography of the energy landscape. For the unmethylated system, the substructure in the main funnel only consists of small, shallow subfunnels. In the modified system, the subfunnels containing structures with the in-configuration are more clearly separated. This separation arises from alterations in the lower stem loop. When considering a transition from $A^{*}$ to $C^{*}$, the changes in the lower and upper stem loop therefore need to be treated as two distinct, but connected events. Considering a lowest minimum to lowest minimum transition $\left(A^{*}\right.$ to $C^{*}$ in the disconnectivity graph), we still observe a significant bias towards the in-configuration, although the equilibrium constant is three orders of magnitude smaller $\left(6.41 \cdot 10^{6}\right.$ compared to $9.30 \cdot 10^{9}$ ). If we consider a transition including the higher energy minima for the in-configuration $\left(B^{*}\right)$ the forward and backward rates are nearly identical and we compute an equilibrium constant around unity. A key change in both cases 
is a significant slow down in the reaction rate from $C^{*}$ to $A^{*}$ and $B^{*}$ by eight orders of magnitude compared to $C$ to $A$. This result means that the change in relative energies will lead to a significant population of the out-configuration in the A22-N6-methylated system.

Heat capacity curves show structural transitions. The changes in the rate and equilibrium constants will significantly impact the population of the different structural ensembles. A useful way to illustrate these changes, and further link them to an energy scale, is to consider the heat capacity curves for both systems. Peaks in heat capacity curves are associated with phase transitions between different states, and in the case of molecular ensembles maybe interpreted as the transitions between different structural ensembles. Furthermore, the heat capacity is directly linked to the occupation probabilities of different configurations, and each peak in the curve can therefore be analysed in terms of increasing and decreasing occupation probabilities (40). This analysis allows to identify two sets of structures, each one dominant on opposite sides of the phase transition (peak). As we go further from the peak one side, the structure dominant on the other side become less and less likely to be observed, while at the peak the two structures co-exist with equal probability.

In Fig. 3, the heat capacity curve for the unmodified stem loop is shown, alongside representative structures for the transitions obtained by the analysis introduced above. In these schemes, the structures on the left hand side of the arrows are the structures dominant below the peak, while the right hand side structure are the ones observed above. As the calculation of the heat capacity curve requires some approximations, and furthermore our simulations use implicit solvent, the curves should only be interpreted qualitatively, with the most important information coming from the difference between the two curves for the two systems.

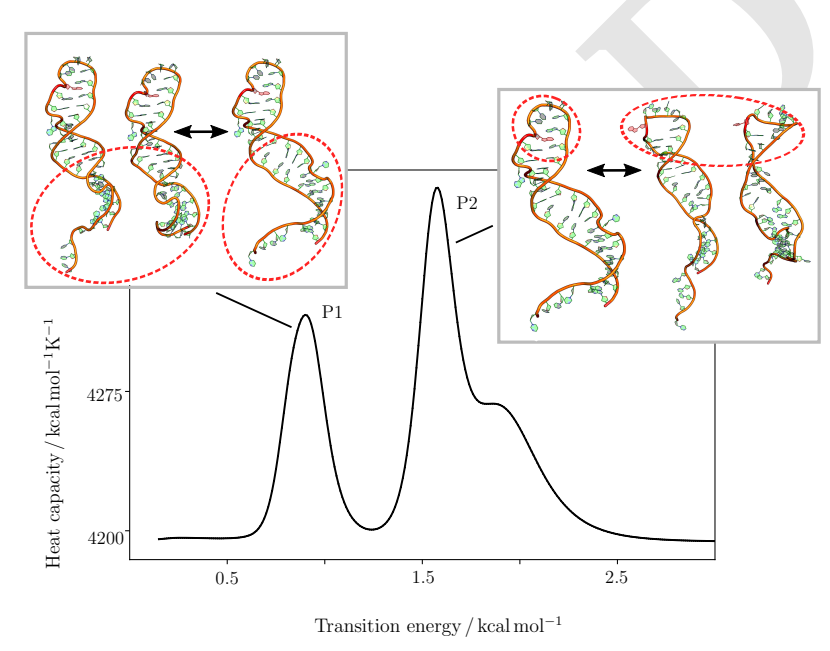

Fig. 3. Heat capacity curve for the unmodified RNA stem loop. Heat capacity curve for the unmethylated RNA stem loop shows three distinct features. Analysing the contributions from local minima to the associated transitions(40) reveals a medium temperature transition between different configurations of the lower stem loop configuration (P1), and a high temperature transition between in- and out-configurations for A23 (P2).

Two important transitions are observed, labelled P1 and P2 in Fig. 3. P1 is the lowest energy feature, which is associated with a rearrangement in the lower stem region. This transition corresponds to higher occupancy of the higher energy structures in the main funnel on the landscape. The second feature, $\mathrm{P} 2$, is the transition from in to out.

We observe the same two transitions for the m6A-modified stem loop, as shown in Fig. 4, also labelled as P1 and P2. Importantly we observe a distinct shift to lower energies for both transitions. We can associated the energy scale for the peaks with Boltzmann population proportions, and find that the P2 peak in the modified system is at energies accessible to around $26 \%$ of molecules, while in the unmodified system this percentage shrinks to only $7 \%$. This shift is the key observation, as the transition associated with P2 is likely required for binding, and is significantly more likely in the modified system.

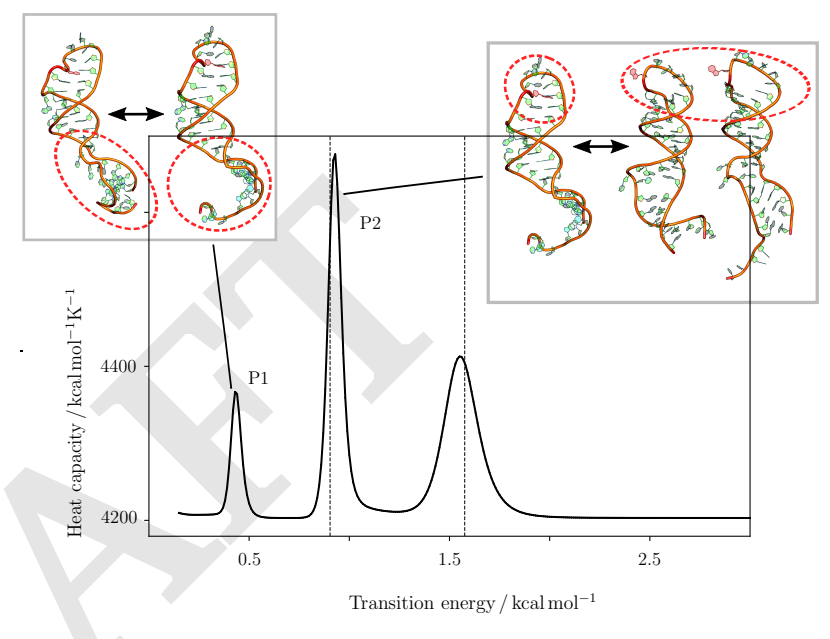

Fig. 4. Heat capacity curve for the A23-methylated RNA stem loop. Heat capacity curve for the A23-methylated RNA stem loop shows three distinct features similar to the unmodified stem loop (see Fig. 3), but the peaks are shifted to lower temperatures. For reference, the peak positions in the unmodified loop are shown as dashed lines. The transition between different configurations of the lower stem loop configuration ( $\mathrm{P} 1$ ) is now at temperatures well below room temperature, and the transition between in- and out-configurations for A23 (P2) at medium temperatures.

Structural variations observed in the energy landscapes. Having established that the m6A-modified stem loop shows significant alterations in the occupation of the structural ensembles on the energy landscape, we will need to look at the structural ensembles more closely to identify the key changes introduced by the N6-methylation of A22, and how these changes alter the transition mechanism from the in- to the out-configuration.

The details of the local behaviour in the four structural ensembles $\mathrm{A}, \mathrm{C}, \mathrm{A}^{*}$ and $\mathrm{C}^{*}$ are given in fig. 5 where we can observe the variations in base pairing (canonical and noncanonical), stacking and pucker configuration for each nucleotide. The first global observation is that the canonical base pairing is well preserved across the four ensembles, with only one or two variations and with A exhibiting the most canonical pairs. Based on these canonical base-pairings we can identify secondary structural elements common to all ensembles. A first helical region is were pairing between nucleotides G4 to A7 with nucleotides U39 to U42 is observed 
Table 1. Rate constants and equilibrium constants between $A, B$ and $C$ at $310 \mathrm{~K}$

\begin{tabular}{c|c|c|c|c} 
System & Set of minima & $k_{\text {out } \leftarrow \text { in }}\left(\mathrm{s}^{-1}\right)$ & $k_{\text {in } \leftarrow \text { out }}\left(\mathrm{s}^{-1}\right)$ & $K_{\text {eq }}$ \\
\hline A23 & $A+B \longleftrightarrow C$ & $1.535 \cdot 10^{-10}$ & 1.427 & $9.30 \cdot 10^{9}$ \\
m6A23 & $A^{*} \longleftrightarrow C^{*}$ & $4.588 \cdot 10^{-15}$ & $2.943 \cdot 10^{-8}$ & $6.41 \cdot 10^{6}$ \\
m6A23 & $B^{*} \longleftrightarrow C^{*}$ & $2.262 \cdot 10^{-8}$ & $2.943 \cdot 10^{-8}$ & 1.30
\end{tabular}

Rate constants and the related equilibrium constants at $310 \mathrm{~K}$ for the unmodified (A23) and the modified (m6A23) system. For the methylation, the two different transitions are given, as described in the full text.

(H1), with an additional base pair between $\mathrm{U} 3$ and A43 often occurring. A second helical region (H2) is more variable, but its core contains nucleotides G13 to $\mathrm{C} 17$ paired with G32 to C37, where either C33 or U34 are not paired. Two other key region are the apical loop (L) sitting above the m6Amodification site containing nucleotides $\mathrm{C} 23$ to $\mathrm{U} 27$, and the bulge in the lower stem (B), which is formed by A8 to C11.

The overall puckering is also well preserved across the ensembles with only a few, but key, transitions from C3'-endo to $\mathrm{C}^{2}$-endo in going from $\mathrm{A}$ to $\mathrm{C}$ and from $\mathrm{A}$ to $\mathrm{A}^{*}$ and $\mathrm{C}^{*}$. These changes involve nucleotides that significantly rearrange from one ensemble to another by either switching basepairing partner or swinging outward or inward with respect to the helical stems. Non-canonical pairing and stacking exhibit more variability across the ensembles with changes spread all across the structure. We can observe a loss of non-canonical pairing in going from $\mathrm{A}$ to $\mathrm{C}$ as well as in going from $\mathrm{A}^{*}$ to $\mathrm{C}^{*}$, and to a lesser extent going from $\mathrm{A}$ to $\mathrm{A}^{*}$. The parameter showing the largest variability is stacking with many significant changes occurring in all parts of the molecule, including the two helical stems and the apical loop.

From all these observations we detail below the most significant structural changes across the four ensembles.

The in-configuration in the unmodified and m6A-modified stem loop. A comparison of the secondary structure of the unmodified and modified stem loop in their lowest energy ensembles ( $A$ and $A^{*}$ ) is shown in Fig. 6 (a), highlighting some important changes. The first alterations observed are located in the upper stem loop in helix H2. A triplet formed between the canonically-paired G16-U34 and C33 is lost in the modified stem loop, and with it the G16-U34 base pair is replaced by a G16-C33 canonical base pairing. Moreover, this change is accompanied by the base pairing of C18-G31. In addition, more non-canonical interaction in the apical loop L are observed, leading to a tightly packed loop, where C23 to U27 are all pointing inwards. Last but not least, the bulge B undergoes significant rearrangement, with U12 and A38 canonically paired, replacing a non-canonical A8-A38 pairing in the unmodified system, which further results in $\mathrm{C} 11$ pointing out alongside C10. Helix $\mathrm{H} 1$ is less affected, and the only change is the existence of the additional non-canonical base pairing between $\mathrm{A} 8$ and $\mathrm{A} 38$ stacked on top of the helical stack.

The changes between them might therefore be summarised as follows. The helical region $\mathrm{H} 2$ is significantly extended in the m6A-modified loop. The stacking in the modified stem loop is altered compared to the unmodified loop (see Fig. 5). This modification is a result of the alteration of A22, such that m6A22-G28 is in a different configuration, with m6A22 sitting somewhat further outside the stacked nucleotides, likely due to the additional space requirement by the methyl group. This alteration allows stacking for G28 and m6A22, with alignment of more nucleotides along $\mathrm{H} 2$, leading to a larger stacked region including more paired bases, albeit at the cost of more strain in the backbone. This extension of the helical region impacts the lower stem, in particular the bulge $\mathrm{B}$, and leads to $\mathrm{C} 11$ pointing outwards. This change in B is very similar to the change in this region observed in the outconfiguration for the unmodified stem loop (see Fig. 6 (b)).

Changing from in- to out-configuration in the unmodified stem loop. The difference between the in- and outconfiguration in the unmodified stem loop is characterised by changes in two main regions: the bulge $\mathrm{B}$ in the lower stem loop illustrated in Fig. 6 (b), and changes in helix $\mathrm{H} 2$ and the apical loop L as shown in Fig. 6 (c). The changes in $\mathrm{B}$ are $\mathrm{C} 11$ pointing outwards, and the non-canonical interaction between $\mathrm{A} 8$ and $\mathrm{A} 38$ being lost. This alteration leads changes in kinking between $\mathrm{H} 1$ and $\mathrm{H} 2$, and associated changes in the backbone. This change in B and at the lower end of $\mathrm{H} 2$ is also observed in higher energy structures with the in-configuration for A22, and can be followed by rearrangements at the top of $\mathrm{H} 2$, which are the loss of the G16-U34 base pair and the formation of the G16-C33 base pair instead. This alternative pairing leads to stacking from this new base pair up to the apical loop on top of C33. The stacking stabilises this configuration in which A22 can swing out. While this leads to a loss of contacts in the apical loop, we do not observe a clear tendency for all nucleotides in this region to change, and the stacking and non-canonical interactions are preserved for $\mathrm{C} 23, \mathrm{U} 24$ and $\mathrm{G} 25$.

Changes in the m6A modified system and the importance of changes in the lower bulge. As described above the changes observed in the lower stem loop for the unmodified system from $A$ to $C$ are largely similar to the changes observed going from the unmodified to the m6A-modified system (i.e. $A$ to $\left.A^{*}\right)$. However, there are two alternative configurations for the bulge region (one observed in $A^{*}$, and the other in $B^{*}$ and $C^{*}$ ). The stem loop is kinking in the bulge in the out-configurations, while in $A^{*}$ there is no kink. In fact, this change in structure is the difference between the ensembles $A^{*}$ and $B^{*}$. The kinking is introduced by stacking between U9 and U12, leading to a significant change in the struc- 

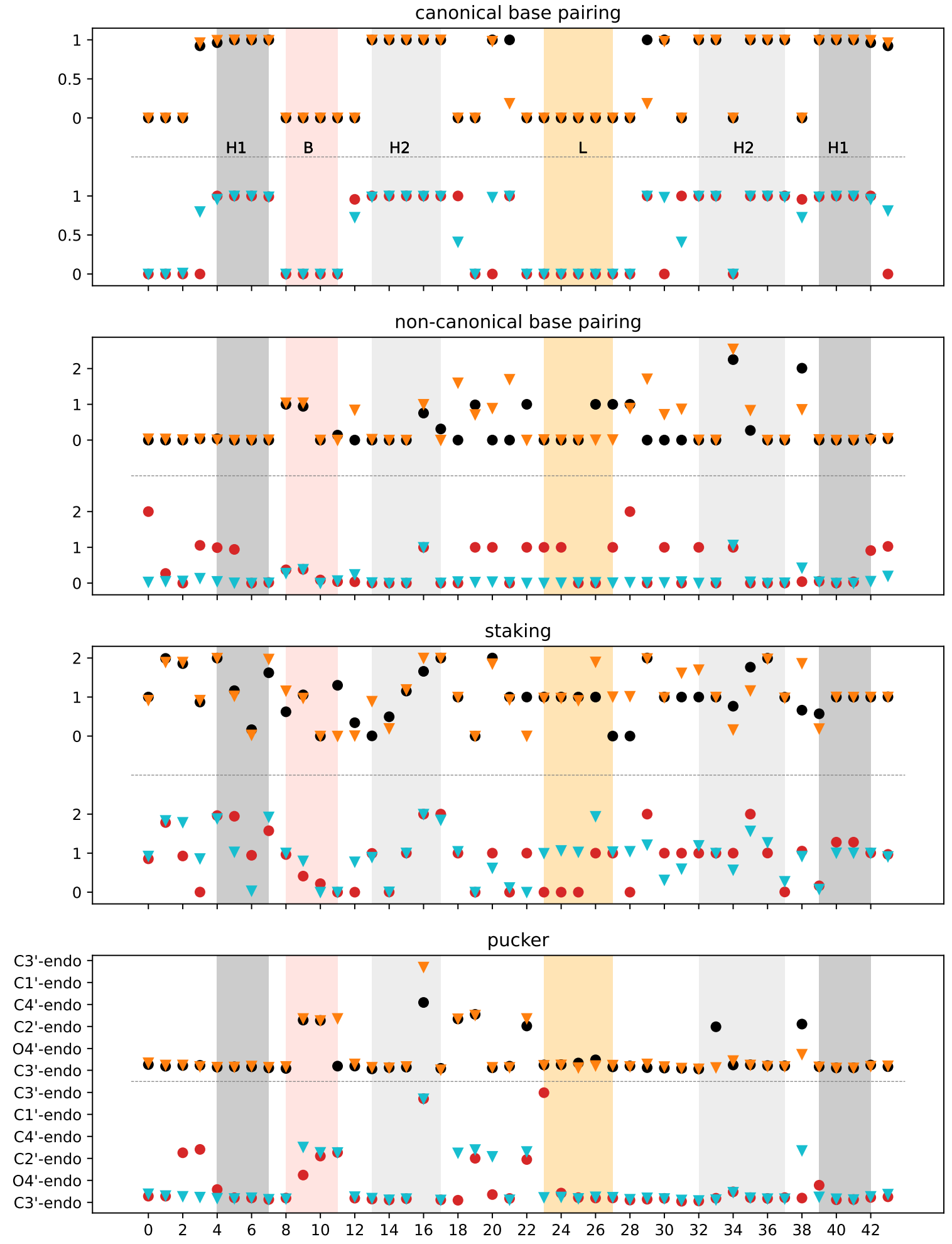

- $A \quad \nabla \quad C \quad$ m6AA $\quad \nabla \quad m 6 A C$

Fig. 5. Local structural properties.

For each of the four low-energy ensembles, $A, C, m 6 A A\left(A^{*}\right)$ and $m 6 A C\left(C^{*}\right)$, we report the behaviour of each nucleotide in terms of number of canonical and non-canonical base pairing, independently of the partners, number stacking interactions formed, and puckering conformation, as extracted from Barbnaba software.

ture, but without the requirement to change other parts of the molecule. The new configuration will be higher in energy due to the introduced backbone strain in the bulge.
The structural changes associated with the transition to the out-configuration in the modified system are more modest than in the unmodified case (see Fig. 6 (d)). Two additional 


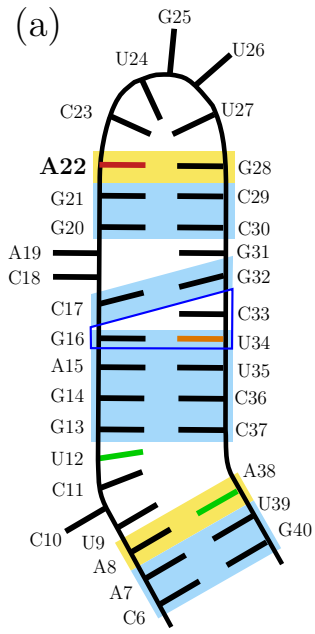

unmodified $A$

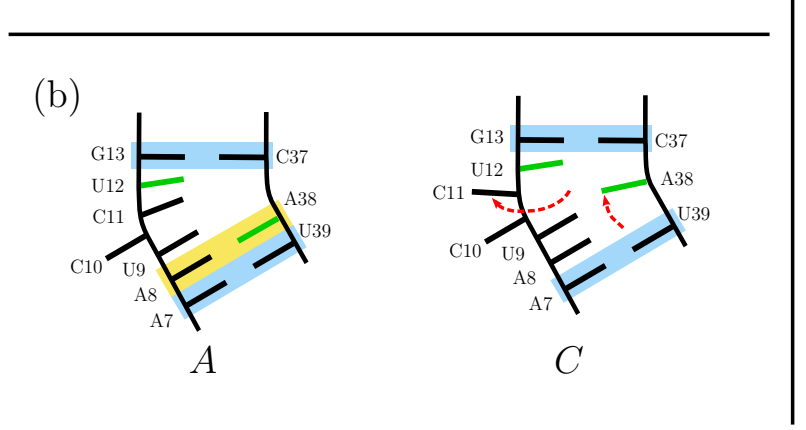

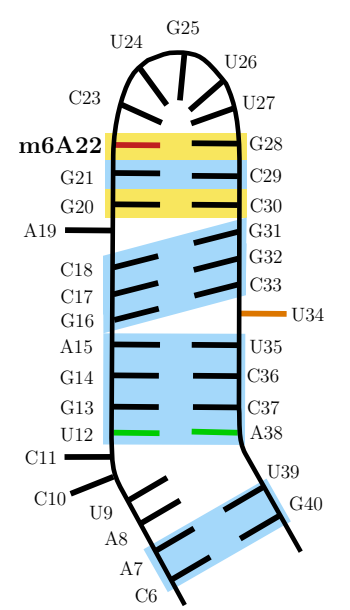

m6A-modified $A$
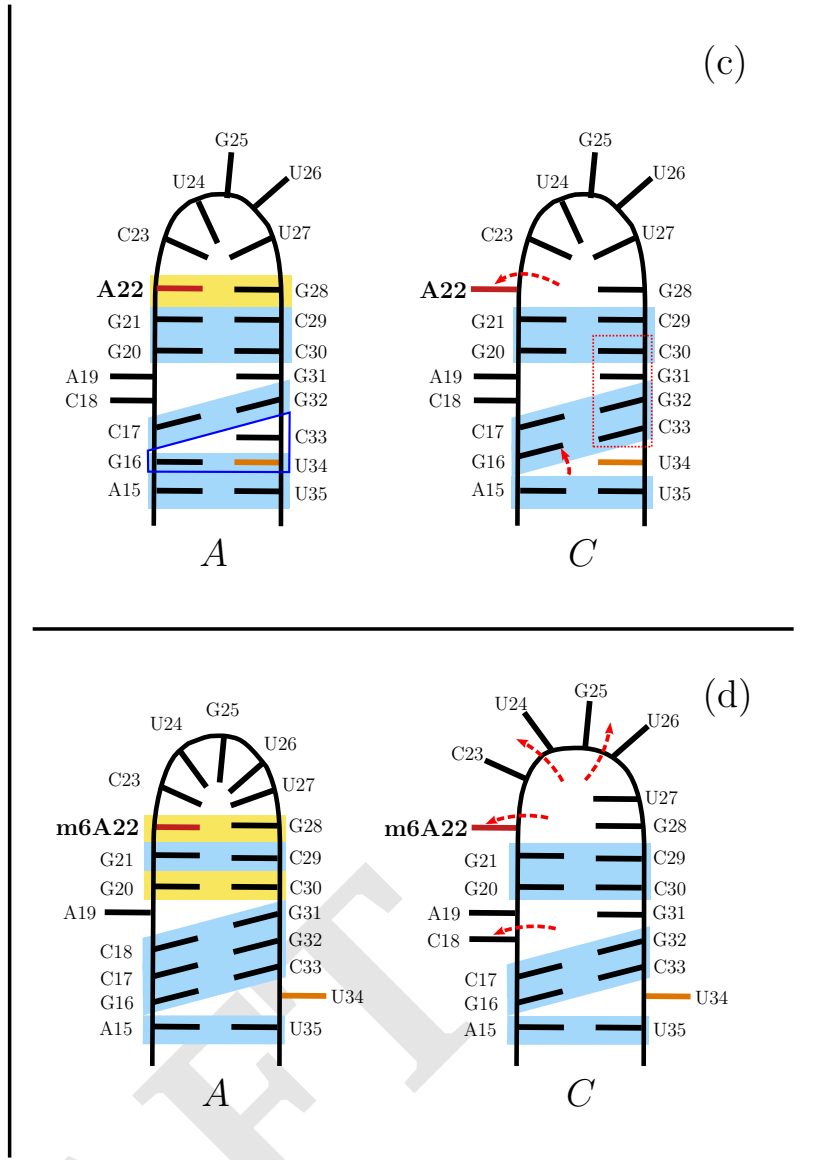

(d)

Fig. 6. Two-dimensional structures for the key structural ensembles. Schematic two-dimensional structures are shown for the key structural ensembles, and their key features are highlighted and compared. Canonical base pairing is indicated in blue, and non-canonical base pairing interactions in yellow. A22 is highlighted in red, and other important residues are also highlighted, namely U34 (orange), U12 and A38 (both green). Key variations between structures are indicated with red dashed arrows, indicating the change in nucleobase orientation. Formed triplets are indicated by a blue frame and important stacking in a red frame. The data used in this figure is derived from the ensemble properties, which are shown in Fig. 5 in more detail. (a) Comparison of the lowest energy ensembles for the unmodified and m6A-modified stem loop. (b) Changes in the bulge between the in-configuration $(A)$ and the out-configuration $(C)$ for the unmodified system. (c) Changes in the upper stem loop between the in-configuration $(A)$ and the out-configuration $(C)$ for the unmodified system. (d) Changes in the upper stem loop going from the in-configuration $\left(A^{*}\right)$ to the out-configuration $\left(C^{*}\right)$ for the m6A-modified stem loop.

changes are observed in going from $A^{*}$ to $C^{*}$ asides from the changes in the bulge discussed. The first one is located in the helical region $\mathrm{H} 2$, where the $\mathrm{C} 18-\mathrm{G} 31$ base pair is lost, changing the interactions within the helix somewhat and leading to some more stabilisation, likely due to the loss of the non-canonical interaction between m6A22 and G28 and the associated stacking. The second change is probably more interesting, as the nucleotides in the apical loop are all swinging out, similar to a fist that is transformed into and open hand, replacing the non-canonical interactions with stacking.

\section{Discussion}

The in/out-transition is too unfavourable in the unmodified system. The first question that needs to be answered with regard to the binding of SND1 to the ORF50 transcript is why this binding is not occurring in absence of the modification. Because no binding in absence of the modification is observed experimentally (6), and our analysis shows the key change is the change in the in/outconfiguration of A22, we are led to the conclusion that the out-configuration is likely associated with binding, and so the accessibility of this configuration is important. While the out-configuration exists in the unmodified stem loop, the change in free energy between the in- and out-configurations compared to the m6A-modified stem loop significantly affects its occupancy. The high stability of the in-configuration and the comparatively low stability of the out-configuration result in a very fast transition from out to in, while the opposite transition is very slow. As a result the life time of the outconfiguration is incredibly short, and the likelihood of transitions to it incredibly small, meaning its population is approximately zero. This observation is supported by the heat capacity curves and equilibrium constants calculated from the free energy landscapes. Hence, the most likely reason no binding is observed in the unmodified case is that the required RNA configurations are simply not available.

Changes in bulge in the lower stem are required for out-configurations. Given these observations, the next question is what is required for a transition to an outconfiguration. A key observation is the absence of any out-configuration without a kinked bulge region, while such 
structures are lowest in free energy for the in-configuration. Likely therefore, the change in the structure lower in the stem is required to allow for changes in the configuration in and around the apical loop, where the GGACU binding motif is located. The large number of unpaired nucleotides in the bulge makes the arrangement of these nucleotides challenging, and generally at least one nucleotide sits outside the stem. The kinking in the bulge region reduces the interactions within the region and is associated with two nucleotides pointing away from the stem loop. However, this loss of interactions is associated with more flexibility and hence entropically favoured. The unkinked bulge is more stable due to the increased interactions, but as the arrangement of the nucleotides is difficult due to the crowding, it locks the stem loop structure. Hence, a change in the upper part of the stem loop is therefore linked to structural changes of the bulge region, allowing for more flexibility in the rest of the stem loop.

m6A22 destabilises the central helix $\mathrm{H} 2$ and alters the bulge structure. This interaction between the apical loop and the bulge through the helical region $\mathrm{H} 2$ naturally leads to the question how the N6-methylation of A22 alters this behaviour. As described in the results section, the methyl group requires more space, and the nucleobase is therefore moving relative to the stacked bases below. m6A22 still forms a noncanonical base pair with G28, where the changed position of the base pair alters the stacking in the helical region all the way down to the bulge. The alteration in the base pairing and stacking interactions lowers the relative stability compared to the unmodified structures in the in-configuration. It further primes the bulge region for the required kinking, due to changes in the helical region. These structural changes establish a connection between the modification and the changes in the lower region of the stem loop. This connection means there is a requirement for the bulge to adopt a different structure to allow for the out-configuration, while the modification in the apical loop affects the bulge region, leading to a connection between these mechanisms. These intertwined mechanisms provide the structural explanation of how the stem loop is destabilised upon the m6A-modification, and how this process is linked to the lower stem loop and the RNA activation process for binding.

At this point, we can draw a comparison to the experimental findings by Baquero-Perez et al. (6), who provide a number of factors impacting the binding of SND1 to the ORF50 RNA transcript. Firstly, experimentally the system is metastable compared to other stem loops, and m6A-modification destabilises the system further. We observe these two features clearly in our physical modelling, namely through the existence of multiple competing structural ensembles and the changes of their relative stability as A22 is N6-methylated. Our modelling provides structural reasons for the destabilisation, and shows how the m6A-modification affects the structure of the stem loop. Furthermore, through the use of altered stem loops, it was highlighted that the lower region of the stem loop, i.e. the bulge is a key feature necessary for binding. Our model shows this link as well, and we can identify the connection of the configurations in the upper and lower parts of the stem loop through changes in stacking propagated by the helical region H2. Finally, we provide an explanation while binding is severely impaired in the unmodified stem loop.

The opening of the apical loop allows for binding. A last comment is reserved for the changes in the apical loop upon adopting the out-configuration. Not only is the change exposing m6A22 to the outside, but also $\mathrm{C} 23$ and $\mathrm{U} 24$, which are both part of the described binding motif GGACU for SND1. In addition, we observe the full opening of the loop, including C25 and U26. These nucleotides form a stack that is exposed, and can likely be recognised by other molecules, including SND1. This feature leads to an appearance of the apical loop like an outstretched hand, inviting interactions.

\section{Conclusions}

We have presented here a full investigation of the energy landscape of a 43mer stem loop of the ORF50 transcript containing a GGACU binding motif. We propose a structural explanation for the experimental observations that the m6A-modified ORF50 transcript binds to its protein partner, SND1, but does not bind if the adenine in position 22 is not N6-methylated. Our study suggests a change in the position of A22 in the methylated system with the base becoming exposed to the solvent in the modified system. This change is interconnected to other important structural modifications occurring in the loop: (a) with the bases of the apical loop also turning toward the solvent, instead of pointing inside the loop in a network of reciprocal interactions, and (b) alterations in the helix (H2) close to the modified nucleotide, which extend all the way to the central bulge separating the two helical regions of the stem-loop. These conclusions from our modelling analysis are in agreement with the experimental evidence suggesting that structural changes have to occur between the methylated and the native system for the binding to occur. In particular, our suggestion that a restructuring of the whole apical portion of the stem-loop (H2 and loop) is needed to accommodate for the methylated nucleotide is in agreement with the observation that the stem-loop is destabilised and is rearranged in the methylated system.

In our analysis we are able to pinpoint the behaviour of key nucleotides undergoing significant structural changes between the two systems. These structural details open up the possibility of performing new experiments targeting specifically these fine structural details (such as chemical probing). If confirmed, our hypothesis would give a full structural picture of the two systems, the relationship between their structures, and their ability to bind. Moreover, the structure proposed for the modified system could be used for further modelling and experimental studies of the ORF50 system in the presence of the protein, or at least of the portion of the protein known to be involved in binding.

On a more general note, we apply here a new method to study the details of chemically modified RNA structures with a focus on the ensembles of possible alternative structures that the system might adopt. With systems as flexible and poly- 
bioRxiv preprint doi: https://doi.org/10.1101/2021.11.16.468829; this version posted November 18, 2021. The copyright holder for this preprint (which was not certified by peer review) is the author/funder, who has granted bioRxiv a license to display the preprint in perpetuity. It is made available under aCC-BY-NC 4.0 International license.

morphic as RNAs, where multiple structures are frequently observed for a given sequence, this approach is key to be able to correctly interpret experimental data and to link structures with experiments, gaining a coherent view of the system's behaviour. We had applied this approach successfully in the past on a system for which several alternative experimental structures were resolved and for which mutational data was available, and in this work we report the first example of a study of the changes of the structural ensembles upon epigenetic modifications without any available experimental threedimensional structures.

Data availability.. The databases containing all minima and transition states and the analysis scripts used are available online (DOI:10.5281/zenodo.5647374).

\section{ACKNOWLEDGEMENTS}

$\mathrm{KR}$ is funded by the Cambridge Philosophical Society. KR and SP are grateful for the visiting fellowship granted to KR by the Faculté de Santé of Université de Paris. AMB is funded by the MRC (MR/R010145/1). AW is funded by MRC (MR/R010145/1) and BBSRC (BB/T00021X/1, BB/V006363/1) project grants. This preprint version of the manuscript is compiled using Ricardo Henriques' LaTeX template for bioRxiv.

\section{Biobliography}

1. Chris Boshoff and Robin Weiss. Aids-related malignancies. Nat. Rev. Cancer, 2(5):373382, 2002.

2. Jonathan Guito and David M. Lukac. Kshv rta promoter specification and viral reactivation. Front. Microbiol., 3:30, 2012.

3. Lucy Dalton-Griffin, Sam J. Wilson, and Paul Kellam. X-box binding protein 1 contributes to induction of the kaposi's sarcoma-associated herpesvirus lytic cycle under hypoxic conditions. J. Virol., 83(14):7202-7209, 2009.

4. David A. Davis, Andrea S. Rinderknecht, J. Paul Zoeteweij, Yoshiyasu Aoki, Elizabeth L. Read-Connole, Giovanna Tosato, Andrew Blauvelt, and Robert Yarchoan. Hypoxia induces Iytic replication of kaposi sarcoma-associated herpesvirus. Blood, 97(10):32443250, 2001.

5. Oliver Manners, Belinda Baquero-Perez, and Adrian Whitehouse. m6a: Widespread regulatory control in virus replication. BBA - Gene Regul. Mech., 1862(3):370-381, 2019.

6. Belinda Baquero-Perez, Agne Antanaviciute, Ivaylo D Yonchev, Ian M Carr, Stuart A Wilson, and Adrian Whitehouse. The tudor snd1 protein is an m6a rna reader essential for replication of kaposi's sarcoma-associated herpesvirus. eLife, 8:e47261, 2019.

7. Hao Du, Ya Zhao, Jinqiu He, Yao Zhang, Hairui Xi, Mofang Liu, Jinbiao Ma, and Ligang Wu Ythdf2 destabilizes m6A-containing rna through direct recruitment of the ccr4-not deadenylase complex. Nat. Commun., 7(1):12626, 2016.

8. Xiao Wang, Zhike Lu, Adrian Gomez, Gary C. Hon, Yanan Yue, Dali Han, Ye Fu, Marc Parisien, Qing Dai, Guifang Jia, Bing Ren, Tao Pan, and Chuan He. N6-methyladenosinedependent regulation of messenger rna stability. Nature, 505(7481):117-120, 2014.

9. Xiao Wang, Boxuan Simen Zhao, Ian A. Roundtree, Zhike Lu, Dali Han, Honghui Ma, Xiaocheng Weng, Kai Chen, Hailing Shi, and Chuan He. N6-methyladenosine modulates messenger rna translation efficiency. Cell, 161(6):1388-1399, 2015.

10. Nian Liu, Qing Dai, Guanqun Zheng, Chuan He, Marc Parisien, and Tao Pan. N6methyladenosine-dependent rna structural switches regulate RNA-protein interactions. $\mathrm{Na}$ ture, 518(7540):560-564, 2015.

11. Nian Liu, Katherine I. Zhou, Marc Parisien, Qing Dai, Luda Diatchenko, and Tao Pan. N6methyladenosine alters rna structure to regulate binding of a low-complexity protein. Nucleic Acids Res., 45(10):6051-6063, 2017.

12. Konstantin Röder, Guillaume Stirnemann, Anne-Catherine Dock-Bregeon, David J. Wales, and Samuela Pasquali. Structural transitions in the rna 7 sk $5^{\prime}$ hairpin and their effect on hexim binding. Nucleic Acids Res., 48:373-389, 2020.

13. Jerelle A. Joseph, Konstantin Röder, Debayan Chakraborty, Rosemary G. Mantell, and David J. Wales. Exploring biomolecular energy landscapes. Chem. Commun., 53(52): 6974-6988, 2017.

14. K. Röder, J. A. Joseph, B. E. Husic, and D. J. Wales. Energy landscapes for proteins: From single funnels to multifunctional systems. Adv. Theory Simul., 2(4):1800175, 2019.

15. K. Röder and S. Pasquali. Rna modelling with the computational energy landscape framework. In L. Ponchon, editor, RNA Scaffolds, volume 2323 of Methods in Molecular Biology, pages 49-66. Humana, New York, 2021.

16. Mariusz Popenda, Marta Szachniuk, Maciej Antczak, Katarzyna J. Purzycka, Piotr Lukasiak, Natalia Bartol, Jacek Blazewicz, and Ryszard W. Adamiak. Automated 3D structure composition for large rnas. Nucleic Acids Res., 40(14):e112, 2012.

17. Z. Li and H. A. Scheraga. Monte Carlo-minimization approach to the multiple-minima problem in protein folding. Proc. Natl. Acad. Sci. USA, 84(19):6611-6615, 1987.

18. Z. Li and H. A. Scheraga. Structure and free-energy of complex thermodynamic systems. J. Mol. Struct., 48:333-352, 1988.

19. David J. Wales and Jonathan P. K. Doye. Global optimization by basin-hopping and the lowest energy structures of Lennard-Jones clusters containing up to 110 atoms. J. Chem. Phys. A, 101(28):5111-5116, 1997.
20. Kenji Mochizuki, Chris S. Whittleston, Sandeep Somani, Halim Kusumaatmaja, and David J. Wales. A conformational factorisation approach for estimating the binding free energies of macromolecules. Phys. Chem. Chem. Phys., 16(7):2842-2853, 2014.

21. Mark T. Oakley and Roy L. Johnston. Energy landscapes and global optimization of selfassembling cyclic peptides. J. Chem. Theory Comput., 10(4):1810-1816, 2014.

22. David J. Wales. Discrete path sampling. Mol. Phys., 100(20):3285-3305, 2002.

23. David J. Wales. Some further applications of discrete path sampling to cluster isomerization. Mol. Phys., 102(9-10):891-908, 2004.

24. Frank Noé and Stefan Fischer. Transition networks for modeling the kinetics of conformational change in macromolecules. Curr. Opin. Struc. Biol., 18(2):154-162, 2008.

25. David J Wales. Energy landscapes: some new horizons. Curr. Opin. Struc. Biol., 20(1): 3-10, 2010.

26. Graeme Henkelman and Hannes Jónsson. Improved tangent estimate in the nudged elastic band method for finding minimum energy paths and saddle points. J. Chem. Phys., 113(22): 9978-9985, 2000.

27. Graeme Henkelman, Blas P. Uberuaga, and Hannes Jónsson. A climbing image nudged elastic band method for finding saddle points and minimum energy paths. J. Chem. Phys., 113(22):9901-9904, 2000.

28. Semen A. Trygubenko and David J. Wales. A doubly nudged elastic band method for finding transition states. J. Chem. Phys., 120(5):2082-2094, 2004.

29. Lindsey J. Munro and David J. Wales. Defect migration in crystalline silicon. Phys. Rev. B, 59(6):3969-3980, 1999.

30. J. M. Carr and D. J. Wales. The energy landscape as a computational tool. In J.-P. Connerade and A. Solov'yov, editors, Latest Advances in Atomic Cluster Collisions: Structure and Dynamics from the Nuclear to the Biological Scale. Imperial College Press, London, 2008.

31. D. J. Wales. Calculating rate constants and committor probabilities for transition networks by graph transformation. J. Chem. Phys., 130:204111, 2009.

32. Oren M. Becker and Martin Karplus. The topology of multidimensional potential energy surfaces: Theory and application to peptide structure and kinetics. J. Chem. Phys., 106(4): 1495-1517, 1997.

33. David J. Wales, Mark A. Miller, and Tiffany R. Walsh. Archetypal energy landscapes. Nature, 394(6695):758-760, 1998

34. Junmei Wang, Piotr Cieplak, and Peter A Kollman. How well does a restrained electrostatic potential (RESP) model perform in calculating conformational energies of organic and biological molecules? J. Comput. Chem., 21(21):1049-1074, 2000.

35. Alberto Pérez, Iván Marchán, Daniel Svozil, Jiri Sponer, Thomas E. Cheatham, Charles A. Laughton, and Modesto Orozco. Refinement of the AMBER force field for nucleic acids: Improving the description of $\alpha / \gamma$ conformers. Biophys. J., 92(11):3817-3829, 2007.

36. Pavel Banáš, Daniel Hollas, Marie Zgarbová, Petr Jurečka, Modesto Orozco, Thomas E. Cheatham, Jiři Šponer, and Michal Otyepka. Performance of molecular mechanics force fields for RNA simulations: Stability of UUCG and GNRA hairpins. J. Chem. Theory Comput., 6(12):3836-3849, 2010.

37. Marie Zgarbová, Michal Otyepka, Jiř́ Šponer, Arnošt Mládek, Pavel Banáš, Thomas E. Cheatham, and Petr Jurečka. Refinement of the Cornell et al. Nucleic acids force field based on reference quantum chemical calculations of glycosidic torsion profiles. J. Chem. Theory Comput., 7(9):2886-2902, 2011.

38. R. Aduri, B. T. Psciuk, P. Saro, H. Taniga, and J. Schlegel, H. B. an SantaLucia. Amber force field parameters for the naturally occurring modified nucleosides in rna. J. Chem. Theory Comput., 3(4):1464-1475, 2007.

39. Sandro Bottaro, Giovanni Bussi, Giovanni Pinamonti, Sabine Reißer, Wouter Boomsma, and Kresten Lindorff-Larsen. Barnaba: software for analysis of nucleic acid structures and trajectories. RNA, 25:219-231, 2019.

40. David J. Wales. Decoding heat capacity features from the energy landscape. Phys. Rev. E, 95:030105, 2017. 